\title{
Características radiográficas das massas incidentalmente descobertas em pacientes posteriormente diagnosticados com câncer adrenocortical
}

\author{
Thiago Machado Nogueira ${ }^{1}$, Roy Lirou ${ }^{2}$, Maria Candida Barisson Villares Fragasso ${ }^{2}$, Tobias Else ${ }^{2}$
}

Nogueira TM, Lirou R, Fragasso MCBV, Else T. Características radiográficas das massas incidentalmente descobertas em pacientes posteriormente diagnosticados com câncer adrenocortical. Rev Med (São Paulo). 2015 jan,-mar.;94(1):57-8.

Tumores adrenais benignos são comuns (5$10 \%$ da população) sendo frequentemente descobertos incidentalmente. Câncer adrenocortical (CAC), por outro lado, é uma neoplasia extremamente rara (incidência de 1/ milhão/ano). O risco de CAC é a principal motivação para o seguimento de incidentalomas adrenais (IA), contudo os benefícios do follow-up com técnicas de imagem não foram estabelecido tendo em vista a sua raridade, havendo controvérsia entre os guidelines sobre seu seguimento (principalmente para IA menores que $6 \mathrm{~cm}$ ).

Este estudo teve como objetivos analisar retrospectivamente casos de IA posteriormente diagnosticados como CAC e definir suas características radiológicas e clínicas.

Foram incluídos no estudo IA $<6 \mathrm{~cm}$ na imagem inicial realizada, no mínimo, 5 meses antes do diagnóstico de CAC. Vinte casos foram identificados em um repositório eletrônico com devido consentimento para a realização de pesquisa acadêmica. As imagens radiológicas e as características clínicas dos tumores foram analisadas. As imagens foram revisadas por dois radiologistas independentes especializados em afecções das adrenais. A razão para a imagem inicial não teve relação com sintomas endócrinos em todos os casos.
A idade média ao achado radiológico inicial foi de $47,1 \pm 16,8$ anos e de 50,2 $\pm 16,1$ anos ao diagnóstico de CAC. O intervalo médio entre achado inicial e diagnóstico de CAC foi de 44,1 \pm 39,5 meses (range: 6-131 meses) com 5 CACs diagnosticados em até 12 meses (25\%) e 10 em até 24 meses $(50 \%)$. O tamanho médio das lesões iniciais foi de $3,2 \mathrm{~cm}$ (mediana $3,4 \mathrm{~cm}$, range: $1,1-5,3 \mathrm{~cm}$ ). Quatorze IA eram $<4 \mathrm{~cm}$ em seu maior diâmetro quando identificados e cinco eram $<2 \mathrm{~cm}$. Nove dos vinte pacientes (45\%) apresentaram evidência bioquímica de hipersecreção hormonal após a identificação do IA ou em algum momento entre o achado inicial e o diagnóstico de CAC.

Entre os 9 tumores funcionantes, cinco produziam cortisol, seis produziam andrógenos e dois produziam aldosterona. Três deles secretavam mais de um tipo de hormônio. A presença de sinais sugestivos de malignidade (heterogeneidade, atenuação $>10 \mathrm{HU}$, margens irregulares, ausência de queda de sinal na ressonância magnética e alto sinal em T2) eram evidentes em todos, exceto dois IA. A taxa de crescimento média do maior diâmetro foi de 1,92 $\pm 1,66 \mathrm{~cm} /$ ano (range: $0,2-6,0 \mathrm{~cm} / \mathrm{ano}$ ).

Em seis casos, um período inicial de latência foi seguido por crescimento acelerado. Nove lesões apresentaram uma taxa de crescimento entre o achado inicial e o diagnóstico inferior a $1 \mathrm{~cm} / \mathrm{ano}$.

$1^{\circ}$ lugar Prêmio Painéis - Área Clínica no 33 COMU - Congresso Médico Universitário da FMUSP, SP, 31 de out. a 02 de nov. de 2014. 1. Acadêmicos de Medicina da Faculdade de Medicina da Universidade de São Paulo.

2. Orientadores, Faculdade de Medicina da Universidade de São Paulo. 
Em resumo, os dados mostram que há grande variabilidade das características iniciais e na evolução dos IA posteriormente diagnosticados como CAC. Dessa forma, o seguimento radiológico dos IA, com duração máxima preconizada de 24 meses segundo os guidelines, não se mostrou efetivo em grande parte dos casos.

Nossos achados permitem questionar as recomendações atuais para o seguimento de IA. Tendo em vista a raridade do $\mathrm{CAC}$, seguimentos radiológicos programados regularmente (como proposto nos guidelines), principalmente para lesões com aspecto benigno, pode não ser justificado considerando a exposição dos pacientes à radiação e o custo global para o sistema de saúde. Estratificação individual do risco ou métodos alternativos de vigilância são necessários. 